\title{
émulations
}

\section{Une critique furtive de l'État social}

\section{Une perspective théorique pour comprendre le non- recours raisonné aux prestations sociales}

Jean-Pierre Tabin et Frédérique Leresche

Émulations - Revue de sciences sociales

2019, Varia, En ligne.

Article disponible à l'adresse suivante

https://ojs.uclouvain.be/index.php/emulations/article/view/tabin

\section{Pour citer cet article}

Jean-Pierre Tabin et Frédérique Leresche, « Une critique furtive de l'État social. Une perspective théorique pour comprendre le non-recours raisonné aux prestations sociales », Émulations, Varia, En ligne. Mise en ligne le 09 octobre 2019. DOI : 10.14428/emulations.varia.026

Distribution électronique : Université catholique de Louvain (Belgique) : ojs.uclouvain.be

(C) Cet article est mis à disposition selon les termes de la Licence Creative Commons Attribution, Pas d'Utilisation Commerciale 4.0 International. http://creativecommons.org/licenses/by-nc/4.0/

Éditeur : Émulations - Revue de sciences sociales / Presses universitaires de Louvain https://ojs.uclouvain.be/index.php/emulations

ISSN électronique : 1784-5734

PUL PRESSES

UNIVERSITAIRES 


\title{
Une critique furtive de l'État social
}

\author{
Une perspective théorique pour comprendre le non- \\ recours raisonné aux prestations sociales
}

\author{
Jean-Pierre Tabin et Frédérique Leresche $^{1}$
}

\begin{abstract}
[Résumé] Dans cet article, nous partons d'un angle mort de la théorie de la construction des problèmes sociaux, celui de la résistance des publics à la définition institutionnalisée des problèmes et de leurs solutions. Nous montrons d'abord que la positivité des droits sociaux pour leurs publics potentiels a un caractère hégémonique. Puis nous examinons les recherches existantes sur le non-recours aux droits sociaux pour indiquer qu'il n'est pas toujours, ou pas seulement dû à des dysfonctionnements administratifs, à un manque d'habileté sociale ou à des processus de stigmatisation sociale, il peut être une réaction au pouvoir de l'État. Ce type de non-recours repose sur une critique que nous qualifions de «furtive » en raison du caractère hégémonique de la positivité des droits sociaux. Comme piste pour l'étudier, nous proposons de mobiliser des outils inspirés des perspectives anthropologiques subalterne, postcoloniale et décoloniale.
\end{abstract}

Mots clés : Non-recours, politique sociale, hégémonie, études subalternes et post/décoloniales, prestations sociales.

[Abstract] In this paper, we start from a blind spot of the construction of social problems theory: the resistance of potential audiences to the institutionalized definition of problems and of their solutions. First, we show the hegemonic character of social rights positivity for their potential audiences. Second, we examine non take-up of social rights research to show that it is not always, or not only due to administrative dysfunctions, lack of social skills or processes of social stigmatization: it can be a reaction to state power. This type of non take-up is based on a "furtive" critic of the institutionalized definition of problems and of their solutions because of the hegemonic nature of social rights positivity. To study it, we propose to mobilize tools inspired by subaltern, postcolonial and decolonial anthropological perspectives.

Keywords: Non take-up, social policy, hegemony, subaltern and post/decolonial studies, social benefits.

\footnotetext{
${ }^{1}$ Haute école de travail social Lausanne, HES-SO, Suisse ; Haute école de travail social Lausanne, HES-SO et Université de Genève, UNIGE, Suisse.
} 


\section{Introduction}

« Les acteurs disposent tous de capacités critiques, ont tous accès, quoique sans doute à des degrés inégaux, à des ressources critiques et les mettent en œuvre de façon quasi-permanente dans le cours ordinaire de la vie sociale. »(Boltanski, $1990: 130$ )

La science sociale a donné un large écho à la théorie de Joseph Gusfield (1980) sur la production des problèmes publics. Dans son ouvrage, The culture of public problems, il explique que ces derniers sont la résultante d'un processus collectif de construction d'un problème et de sa solution. La structure des problèmes publics s'articule selon Gusfield sur trois éléments : la "propriété », qui permet d’identifier quel groupe ou quelle institution a le pouvoir sur le problème ; la " responsabilité causale », qui repose sur une théorie de l'enchaînement de faits qui ont provoqué le problème ; enfin la « responsabilité politique » qui permet de désigner l'instance chargée de résoudre le problème. Gusfield s'intéresse en particulier à la relation entre ces trois aspects de la structure ; s'il insiste sur le fait que les propriétaires du problème essaient souvent de faire endosser les obligations liées à sa résolution par d'autres, il relève que l'État joue un rôle clé pour fixer non seulement ce qu'est le problème public, mais encore la solution à ce problème public. Comme l'ont fait remarquer Claude Gilbert et Emmanuel Henry (2009) des rapports de force vont faire que certaines définitions seront privilégiées au détriment d'autres.

Dans le champ des politiques sociales, cette approche est tout à fait heuristique. En ce qui concerne le chômage par exemple, la démarche de Gusfield permet d'identifier quel groupe d'acteurs a réussi à prendre le problème en main. Selon Christian Topalov (1999), ce sont au tournant du XX ${ }^{\mathrm{e}}$ siècle différents notables qu'il a nommé les « réformateurs du nouveau siècle». Jusqu'à cette époque en effet, les problèmes sociaux étaient essentiellement pensés comme individuels, découlant d'une incapacité personnelle ou d'une disposition problématique en regard de la norme de l'emploi ; l'invention de la catégorie « chômage », qui définit les personnes sans emploi désireuses et capables d'en trouver un, vient rompre avec cette représentation en attribuant à certaines situations problématiques une cause extra-individuelle (Tabin, Togni, 2013). Ces réformateurs ont en effet réussi à imposer comme responsabilité causale du chômage non pas l'individu, mais le caractère irrégulier de la demande industrielle de main-d'œuvre (Beveridge, 1909). Ils ont également pu convaincre que la responsabilité politique était celle de l'État (Salais, Baverez, Reynaud, 1986; Topalov, 1994). De nos jours, la polémique récurrente sur le « vrai » nombre de personnes sans emploi montre que la notion de chômage n'est plus considérée comme une invention de réformateurs, elle est «fermement intégrée dans le réseau des représentations communes » (Desrosières, 1993 : 407).

Lorsque le problème social et sa solution sont constitués, ils sont institutionnalisés dans des dispositifs comme l'assurance chômage, pour reprendre l'exemple cité ci- 
dessus. Ces institutions sont des êtres « sans corps à qui est déléguée la tâche de dire ce qu'il en est de ce qui est » (Boltanski, 2009: 117). Elles énoncent la réalité en matière de problèmes sociaux et ce faisant contribuent à produire - et à transformer - cette réalité. La perspective proposée par Gusfield qui se focalise sur la « carrière des problèmes publics » (Gilbert, Henry, 2012: 36) permet en ce sens de lutter contre ce que Pierre Bourdieu (2012) nomme l'« amnésie de la genèse qui est inhérente à toute institutionnalisation réussie, toute institution qui réussit à s'imposer impliquant l'oubli de sa genèse » (p. 185). Comme l'ont fait remarquer Gilbert et Henry (2012) l'approche du problème public de Gusfield tend à faire «oublier que celui-ci n'est qu'une modalité de définition particulière d'une situation ou d'une condition sociale » (p. 37) et qu'elle est l'objet de luttes définitionnelles. L'analyse de ces processus permet de comprendre pourquoi les sociétés «s'accommodent de certains problèmes parfois très dommageables à certains groupes sociaux et comment se construit la légitimation de l'action publique » (Gilbert, Henry, 2012 : 56). La théorie de Gusfield a également un angle mort qui fait l'objet de cet article. En effet, en se focalisant sur la construction des problèmes publics, elle tend à occulter le fait que même lorsqu'elle est objectivée par l'État qui est le " géométral de toutes les perspectives » (Bourdieu, 2012 : 53), la manière institutionnalisée de construire et de traiter un « problème social » provoque des résistances. Pour reprendre notre exemple, ce n'est pas parce que le « problème du chômage » est intégré dans les représentations sociales que tous les individus ayant perdu leur emploi vont s'y reconnaître ou faire systématiquement appel à la solution proposée par l'État pour le résoudre (l'assurance chômage).

Dans cet article, nous allons développer une perspective théorique pour permettre d'entendre la critique (Boltanski, 2009) qui sous-tend certaines formes de non-recours à l'État social'2. Notre argumentaire tient en trois parties. D’abord, nous montrons que dans le contexte actuel, les droits sociaux sont de manière hégémonique considérés comme positifs pour leurs publics potentiels, ce qui signifie qu'il est très difficile pour ces derniers d'articuler et de faire entendre une critique. Ensuite, nous indiquons sur la base de travaux récents sur le non-recours aux prestations sociales que ce dernier n'est pas seulement la conséquence d'un manque d'information, ou de la crainte d'une stigmatisation, mais qu'il découle également parfois d'une résistance et d'une critique du pouvoir. Enfin, étant donné la positivité hégémonique des droits sociaux, nous défendons l'idée qu'une possibilité heuristique pour l'entendre est de mettre en œuvre une anthropologie inspirée des perspectives subalterne, postcoloniale et décoloniale.

\footnotetext{
${ }^{2}$ Cet article est issu d'une recherche menée dans le cadre du Pôle de recherche national LIVES - Surmonter la vulnérabilité, perspective du parcours de vie. En ligne, consulté le 9 septembre 2019, URL : www.lives-nccr.ch. Merci au comité éditorial de la revue Émulations ainsi qu'aux personnes qui ont réalisé l'évaluation avec des commentaires stimulants.
} 


\section{Le caractère hégémonique de la positivité des droits sociaux}

Les politiques sociales sont pensées comme des réponses à des problèmes sociaux qu'elles participent à objectiver et à institutionnaliser. De manière générale, elles sont considérées comme avantageuses pour leurs destinataires potentiel-le·s. Cette idée a été soutenue dès les origines aussi bien par Otto Bismarck qui voyait dans l'assurance sociale un moyen d'aider les pauvres durant et après leur vie active (Kott, 2003), que par William Beveridge (1909) qui, plus de 30 ans avant qu'il n'expose son fameux plan de sécurité sociale, présentait les politiques sociales comme des réponses à des besoins, notamment à ceux des hommes pour qu'ils puissent jouer leur rôle de gagne-pain.

Sur le plan politique, les partis de gauche ont tout au long du $\mathrm{XX}^{\mathrm{e}}$ siècle confirmé cette représentation des choses en soutenant le développement de politiques sociales, car ils y voyaient un instrument de redistribution des richesses (Korpi, 2006). Si les partis représentant la bourgeoisie tendaient à les limiter au nom du fait qu'elles soutiennent une solidarité collective au détriment de mérites individuels (Rosanvallon, 1981), ils les considéraient également comme avantageuses pour leurs publics. L'Organisation internationale du travail, en fixant toute une série de risques sociaux (maladie, accidents, chômage, vieillesse, invalidité, etc.) auxquels les politiques sociales se doivent de répondre, a confirmé la nature fondamentalement positive des politiques sociales pour leurs publics (Convention n 102 adoptée en 1952 et ratifiée par 58 pays en $2019^{3}$ ). Les constitutions des États soutiennent fréquemment cette représentation, à l'exemple de la constitution helvétique de 1999 qui donne des « buts sociaux » à l'État et qui a inscrit en son article 12 l'idée que les prestations sociales permettent de maintenir ou de soutenir la « dignité humaine ». Dans tous ces cas, la conception explicite ou sousjacente est que les droits sociaux sont favorables à leurs publics potentiels, même s'ils peuvent risquer de réduire leur esprit d'entreprise. Si l'on adopte ce point de vue, les publics en puissance des politiques sociales n'ont pas de raison de les remettre fondamentalement en question, puisqu'elles seraient à leur avantage.

Cette positivité générale du droit social, qui tend à faire oublier qu'il favorise certaines catégories au détriment d'autres, par exemple les autochtones par rapport aux personnes de nationalité étrangère (Tabin, 1999) ou les hommes par rapport aux femmes (Lewis, 1992, 1997 ; Sainsbury, 1999 ; Togni, 2015), est également souvent soutenue par la recherche. Traditionnellement, les politiques sociales sont présentées comme une extension de la citoyenneté civile et politique à une citoyenneté sociale (Marshall, 1996). Les perspectives classiques sur la politique sociale, de Richard Titmuss (1969, 1974) à Gøsta Esping-Andersen (1990 ou 1999), en passant par François-Xavier Merrien (2007) ou encore Jean-Claude Barbier et Marie-Thérèse Letablier (2008), pour n'en citer que quelques-unes, comme les ouvrages de référence sur les politiques sociales ou sur l'État social (par exemple Castles et al., 2010 ; Pierson, Castles, Naumann,

${ }^{3}$ En ligne, consulté le 9 septembre 2019, URL :

www.ilo.org/dyn/normlex/fr/f?p=1000:11300:0::NO:11300:P11300_INSTRUMENT_ID:312247 
2014), partent de l'idée qu'elles sont un élément positif de régulation des sociétés industrielles et postindustrielles et qu'elles amènent, à des degrés divers selon le type d'État social, de la redistribution et de la solidarité. Pierre Bourdieu (1993, 2012) lui-même parle des politiques sociales comme de la « main gauche de l'État », celle qui redistribue l'argent public. Même les scientifiques plus radicaux qui comme Frances Fox Piven et Richard Cloward (1971) dénoncent le contrôle social que soutiennent les politiques sociales, partent de l'idée qu'elles sont favorables aux classes dominées, car leurs analyses reposent sur le postulat qu'elles permettent de diminuer les revendications sociales : les prestations sont vues comme positives pour leur public puisqu'elles vont permettre de « calmer le jobard », pour reprendre l'expression d'Erving Goffman (1952).

Les politiques sociales sont donc considérées comme positives parce que dans leur définition la plus courante elles constituent « un ensemble de mécanismes publics visant à protéger les citoyens » (Ramaux, 2007: 16). Dans les pays qui ont développé des politiques sociales, l'idée qu'il y a des problèmes sociaux et que les solutions se trouvent dans des politiques sociales est donc largement partagée. Il y a dès lors un « accord sur les points de désaccords » (Bourdieu, 2012) : si aujourd'hui les controverses sont parfois vives sur tel ou tel aspect d'un problème social, ou sur la nature, voire sur l'ampleur de la politique sociale à développer, elles ne remettent plus en question l'idée que la solution aux problèmes sociaux se trouve dans des politiques sociales. C'est ce qui nous amène à penser que dans le contexte contemporain, la positivité des droits sociaux pour leurs publics potentiels a un caractère hégémonique.

Le concept d'hégémonie a été utilisé aussi bien par Antonio Gramsci (1978-1996) que par Michel Foucault (1975, 2001, 2004). Si la normalisation des conduites est un trait commun de ces deux approches de l'hégémonie, elle prend un sens bien différent suivant l'un ou l'autre. Gramsci a développé ce concept pour faire ressortir que « la Révolution en Russie ne supposait que la conquête des structures de domination (administration, armée, police), qui constituaient tout l'État à elles seules [tandis qu']en Occident, la révolution doit l'emporter aussi sur l’hégémonie idéologique et sociale exercée par les élites sur la société civile, qui est elle-même au fondement du corps politique » (Pouchepadass, 2000 : 166). Selon sa perspective, l’hégémonie crée une relation de consentement entre groupe dominant et groupes subordonnés (Smart, 1989). Pour Foucault au contraire, et c'est également notre perspective, l'hégémonie est la conséquence de toutes sortes d'opérations engageant des formations discursives et des pratiques institutionnelles qui ne sont pas uniquement liées au groupe dominant. Pour lui, et contrairement à ce que proposait Gramsci, « what gives coherence to modem domination is not merely the perpetuation of capitalist hegemony in the final analysis, but the existence of a shared epistemic core that seeks to normalize and discipline members of society at each domain of social interaction » (Can Gürcan, Bakmer, 2015: 133). La construction et l'institutionnalisation de problèmes sociaux, plus précisément leur normalisation, est une opération de cet ordre.

En résumé, la positivité du droit social pour ses publics potentiels est aujourd'hui une représentation hégémonique. Toutes sortes de formations discursives, notamment scientifiques, et des pratiques institutionnelles le confirment, auxquelles on peut 
encore ajouter les nombreux discours sur l'abus de prestations sociales (Dubois, 2012; Frauenfelder, Togni, 2007) qui reposent sur le postulat que les prestations sociales sont à ce point intéressantes que des personnes chercheraient indûment à les obtenir. Pourtant, le non-recours aux prestations sociales existe, et comme nous allons le voir il peut dans certains cas être considéré comme un acte de résistance à cette représentation hégémonique.

\section{Le non-recours comme résistance}

La recherche sur le non-recours aux prestations sociales s'est remarquablement développée ces dernières années, notamment sous l'impulsion de chercheurs comme Wim Van Oorschot et Philippe Warin (pour une revue critique de la littérature à partir des cadres d'analyse de Van Oorschot et Warin, voire le récent article de Leresche, 2019). Elle a permis de mettre au jour l'importance de ce non-recours qui concerne, selon les années, les dispositifs et les pays étudiés, entre $19 \%$ et plus de $70 \%$ des destinataires potentiels (Dubois, Ludwinek, 2015). La dernière étude menée dans le pays où nous travaillons (la Suisse) a porté sur l'aide sociale dans le canton de Berne et avance le chiffre de 26,3\% de non-recours (Hümbelin, 2016).

Le constat tiré de ces recherches est qu'une partie importante des droits sociaux n'atteint pas son public potentiel, ce qui questionne l'efficacité et l'efficience des politiques sociales. Relevons que les scientifiques qui travaillent sur le non-recours partent généralement du postulat que les politiques sociales sont intéressantes pour leurs destinataires, certains soutenant même en ce sens que ce non-recours pourrait avoir à terme d'importants coûts sociaux parce que les problèmes non résolus vont empirer (Knüsel, Colombo, 2014).

Les travaux de Wim Van Oorschot $(1991 ; 1994 ; 1995$; 1996), principalement réalisés dans la dernière décennie du $\mathrm{XX}^{\mathrm{e}}$ siècle, se centrent sur les individus qui ne font pas valoir leurs droits aux prestations sociales. Ils ont permis de décrire le processus complexe qui conduit au non-recours. Ils montrent que ce dernier découle principalement de leur caractère stigmatisant, d'une administration problématique des prestations et d'une mauvaise connaissance des dispositifs. Autrement dit, dans cette perspective, le non-recours découle de processus complexes liés aux habiletés sociales des individus, à l'État et au climat normatif des sociétés. On peut déduire de ces analyses que le nonrecours ne s'explique pas par un calcul rationnel (de type coûts/bénéfices) qui supposerait que les personnes puissent avoir une stratégie au sens que donne Michel de Certeau (1990) à ce terme. Pour mettre en œuvre une stratégie, il faudrait en effet que le ou la récipiendaire potentielle ait une possibilité de distance - comme un général sur un champ de bataille, ainsi que l'écrit de Certeau - par rapport à l'objectif à atteindre, et qu'elle soit en mesure de faire des choix informés, ce qui ne semble pas être le cas.

Les travaux plus récents de Philippe Warin $(2006$; 2010 ; 2012 ; 2016) et de l'Observatoire des non-recours aux droits et services (Odenore) ont comme caractéristique de 
s'interroger sur l'effectivité de l'offre en matière de prestations sociales partant du constat qu' « aucune offre n'a de public contraint et captif » (Warin, 2006: 101). Ils permettent de saisir que les différents types de non-recours n'ont ni les mêmes causes, ni les mêmes conséquences pour les individus. Ces travaux mettent également en avant des difficultés liées à l'administration des prestations, par exemple lorsqu'elles ne sont pas proposées à leur public potentiel, ou lorsqu'elles sont demandées, mais qu'elles ne sont pas obtenues, ainsi que les problèmes liés à la mauvaise connaissance des dispositifs. Ces travaux sont repris dans la presse sur le mode de la scandalisation ${ }^{4}$, ce qui confirme que les droits sociaux sont vus comme positifs (puisqu'il est considéré comme scandaleux que des personnes n'exercent pas leur droit).

Les chercheur.e.s de l'Odenore analysent en outre un type de non-recours qui n'a pas été traité en profondeur par Van Oorschot : la non-demande. Cette non-demande peut découler du fait que les droits sociaux mettent en danger d'autres droits, comme le droit de séjour et de travail ${ }^{5}$. Mais elle peut également venir du fait que des personnes ne se sentent pas concernées par des politiques sociales, ce qu'elles affirment parfois de manière active (Vial, 2016), ou parce qu'elles ne veulent pas faire usage de leurs droits sociaux. Dans un article récemment publié, Warin (2018) différencie la non-demande subie (due à un manque de connaissances, de savoir-faire, de confiance, de soutien, etc.) de la non-demande intentionnelle qui peut selon lui découler d'un calcul coûtavantage, d'un désaccord avec la norme, d'une préférence pour des alternatives ou encore d'une forme de civisme. La non-demande dans ce dernier cas questionne l'adéquation de l'offre avec son public potentiel, autrement dit «son sens pour les (non) usagers » et doit être envisagé comme "un rapport social à l'offre publique et aux institutions qui la servent» (Warin, 2010: 5). Avec la non-demande, «la légitimité même des politiques devient un objet de débat » (Warin, 2016: 8). Comme on le voit, la dimension proprement politique de ce type de non-recours est mise en avant.

Un des intérêts de l'analyse de cette forme de non-recours, que nous préférons, pour rendre compte de l'agentivité qui la motive, nommer non-recours « raisonné ", est de porter la focale non plus sur les institutions, mais sur l'expérience que font les

\footnotetext{
${ }^{4}$ Cf. par exemple le quotidien Libération du 13 novembre 2012 (« Le non-recours aux droits, un scandale social ») ou du 22 juin 2018 (« Non-recours, des milliards non-distribués »), ou encore Le Monde du 12 juin 2018 («Non-recours : des aides sociales qui n'atteignent pas leurs bénéficiaires $»)$.

${ }^{5}$ Par exemple, la loi fédérale suisse sur les étrangers prévoit ainsi que l'autorisation de courte durée (Permis L) ou une autorisation de séjour (Permis B) peut être révoquée si son titulaire « ou une personne dont il a la charge dépend de l'aide sociale » (art. 62 let. e LEtr). L'autorisation d'établissement d'un étranger peut également être révoquée si cette personne «ou une personne dont il a la charge dépend durablement et dans une large mesure de l'aide sociale » (art. 63 al. 1 let. c LEtr).

${ }^{6}$ Nous ne retenons pas l'expression «non-demande » parce qu'elle décrit l'agentivité par la négative; nous ne retenons pas non plus l'expression «non-recours intentionnel » parce qu'elle postule que le non-recours est focalisé sur un but.
} 
personnes de droits sociaux (Pélisse, 2005). L'analyse du non-recours raisonné permet dès lors de s'intéresser, à l'instar des travaux d'Austin Sarat (1990) sur le welfare, à l'idée du droit que les publics potentiels des dispositifs se font, sachant que ce droit leur est imposé, qui plus est de manière hégémonique comme quelque chose de positif, alors qu'ils n'ont pas l'impression d'avoir participé à son élaboration et qu'ils n'ont aucun pouvoir pour l'interpréter. C'est ce que précise Sarat :

The law that the welfare poor confront is neither a law of reason and justification nor of sacred texts and shared normative commitments. [Recipients] are [...] "caught" inside law's rules but are, at the same time, excluded from its interpretive community. In all of their dealings with welfare they act on "a terrain imposed... and organized by the law of a foreign power" [...] Their law is a law of power and of compulsion, and their experience of being inside, but yet excluded, is one indication of the way that power is exercised over the welfare poor » (Sarat, 1990 : 345 sq.).

Cela signifie que :

the welfare poor construct a consciousness of law on the basis of their daily deprivation, their experience of unequal, often demeaning treatment, and their search for tools with which to cope with an often unresponsive welfare bureaucracy. Law is, for the welfare poor, embodied in a particular set of lived conditions; theirs is a law of practices, not promises, of material transactions, not abstract ideals. (Sarat, 1990 : 378

Comme cet auteur le signale, dans ce contexte de pouvoir, la seule manière de résister est de mettre en œuvre des «tactiques» qui «misent sur une habile utilisation du temps, des occasions qu'il présente et aussi des jeux qu'il introduit dans les fondations d'un pouvoir» (de Certeau, 1990 : 63). Jérôme Pélisse (2005), faisant référence au livre de Patricia Ewick et de Susan Silbey (1998), relève que cette résistance apparaît lorsque les personnes ont l'impression d'être capturées « par le droit [...] comme un pouvoir arbitraire contre lequel elles se sentent impuissantes » (p. 124). Pour ces publics, il n'y a ni subordination totale, ni contestation franche, c'est plutôt d'une forme d'esquive qu'il s'agit : "Foucault used the word "esquiver", a fencing term meaning to dodge or feint, to describe [these] forms of resistance [...]. These tactics are a sort of anti-discipline, which, like the disciplinary power they oppose, are dispersed and invisibly distributed throughout everyday life » (Ewick, Silbey, 1992 : 748). Pour ces raisons, nous pensons que de manière générale le non-recours raisonné ne repose pas sur une critique claire ${ }^{7}$, mais furtive des politiques sociales.

Résumons ce que nous avons examiné jusqu'ici : le non-recours aux prestations sociales n'est pas toujours, ou pas seulement, dû à des dysfonctionnements administratifs, à un manque d'habileté sociale ou à des processus de stigmatisation sociale, il peut

\footnotetext{
${ }^{7}$ Si des prises de position politiques se développent pour répondre au non-recours (et y remédier), aucune n'offre aux publics visés par les droits sociaux un support argumentatif construit sur lequel ils pourraient s'appuyer pour élaborer une critique ouverte.
} 
être une réaction au pouvoir de l'État. Il ne repose pas en ce cas sur une stratégie, mais sur des tactiques qui prennent forme dans des espaces régis par des règles légales et administratives qui échappent souvent aux publics visés par les politiques sociales, avec des variables que des circonstances particulières leur imposent, et dans un contexte où la positivité des droits sociaux pour leurs destinataires potentiels est hégémonique. Ces tactiques sont dès lors, des «manières de faire [...], des style[s], [...] dans un champ qui les régule à un premier niveau » (de Certeau, 1990 : 51). Un élément qui le confirme est que de multiples catégories de non-recours ont été identifiées par la recherche qui montre qu'il est souvent partiel, temporaire et ponctuel (Leresche, 2019). Sur la base de ce que nous avons présenté jusqu'ici, nous soutenons que le non-recours raisonné est un acte subreptice de résistance au pouvoir reposant sur une critique furtive des politiques sociales institutionnalisées.

\section{Comment entendre une critique furtive ?}

Cette critique est furtive parce qu'elle est, comme on l'a vu, à la fois difficile à formuler et à percevoir. Elle n'est dès lors pas simple à repérer. La difficulté empirique qui se pose est double. D'une part, le non-recours raisonné n'est guère pensé socialement comme une critique ou une résistance potentielle aux droits sociaux, ce qui a pour conséquence que la non-demande raisonnée de droits sociaux est le plus souvent « inaudible » comme l'écrit Pierre Mazet (2010). À propos des pratiques françaises du droit à réparation en maladie professionnelle par des personnes atteintes de cancer, Anne Marchand (2016) a fait remarquer, que si les facteurs de non-recours sont très divers : les pouvoirs publics restent sourds à ce qui peut faire obstacle à l'accès au droit à réparation : le contexte (de maladie), les priorités (de vie) des requérants, leurs impossibilités à reconstituer leur parcours d'exposition aux cancérogènes (pas de mémoire institutionnalisée des expositions), les obstacles institutionnels qu'ils doivent affronter et la violence que peut représenter la reconnaissance en maladie professionnelle quand elle est réduite à sa forme indemnitaire (p. 124-125).

Cette surdité est particulièrement marquée quand le droit social témoigne d'un accommodement de fait aux causes qui produisent les situations problématiques dans lesquelles les personnes se trouvent (Marichalar, 2017).

D’autre part, pour les publics potentiels eux-mêmes, le contexte hégémonique de la positivité des droits sociaux "make it exceedingly difficult to think or act in critical ways » (Schulzke, 2015 : 65), ce qui signifie qu'il est extrêmement difficile d'élaborer une pensée critique répondant aux canons classiques de l'argumentation et de la rationalité. Nous partons dès lors de l'idée que le non-recours raisonné est de l'ordre de ce que Michel Foucault dans son cours au Collège de France du 7 janvier 1976 nommait un « savoir assujetti ». Il référait à :

«toute une série de savoirs qui se trouvaient disqualifiés comme savoirs non conceptuels, comme savoirs insuffisamment élaborés: savoirs naïfs, savoirs 
hiérarchiquement inférieurs, savoirs en dessous du niveau de la connaissance ou de la scientificité requises [...] ce savoir que j'appellerais, si vous voulez, le "savoir des gens" (et qui n'est pas du tout un savoir commun, un bon sens, mais au contraire, un savoir particulier, un savoir local, régional, un savoir différentiel, incapable d'unanimité et qui ne doit sa force qu'au tranchant qu'il oppose à tous ceux qui l'entourent). » (Foucault, $1997:$ 9)

Le non-recours raisonné, qui est selon la recherche souvent partiel, temporaire et ponctuel, et qui ne s'exprime pas, ou pas de manière entendable, comme une critique des droits sociaux, est de cet ordre.

Pour répondre à cette double difficulté, nous proposons de mobiliser des outils empiriques développés dans d'autres contextes et qui reposent sur les notions gramscienne ou foucaldienne de l'hégémonie. Il nous semble que les perspectives subalterne, post et décoloniale en fournissent quelques-uns qui permettent d'entendre et de rendre compte des actes de résistance des individus (Stone-Mediatore, 2003). Dans cette dernière partie, nous allons donc esquisser l'usage qui pourrait être fait de ces perspectives pour l'étude du non-recours raisonné aux droits sociaux.

"Ce qui définit les subalternes (notion empruntée au Gramsci des Cahiers de prison), c'est la relation de subordination dans laquelle les élites les tiennent, relation qui se décline en termes de classe, de caste, de sexe, de race, de langue et de culture.» (Pouchepadass, 2000 : 165) Rappelons brièvement d'abord que la perspective subalterne a consisté, sous l'impulsion de Ranajit Guha (1982) et d'autres scientifiques, à "produire une histoire [de l'Asie du Sud] qui restaure [dans une perspective plutôt gramscienne de l'hégémonie] la parole du peuple des "subalternes" et témoigne de sa culture politique autonome, non "prépolitique" ou arriérée mais différente de celle de l'élite » (Pouchepadass, 2004 : 68). Comme le relève Jacques Pouchepadass (2000), il s'agissait d'abord d'« en finir avec l'élitisme de l'historiographie colonialiste, nationaliste et marxiste, qui présentait la résistance populaire à la colonisation et l'épopée du mouvement d'indépendance comme le résultat d'un processus de mobilisation par le haut» (p. 163). Cette perspective fait clairement partie du courant historiographique qu'il est «convenu d'appeler "l'histoire par le bas" ( (Pouchepadass, 2000 : 162). Elle s'inscrit donc dans le prolongement des travaux d'Edward P. Thomson - notamment sur les économies morales - qui avait pour projet de reconnaître aux "pauvres » des compétences sociales (Fassin, 2009). Elle vise à rétablir «le peuple comme sujet de sa propre histoire en refusant de le concevoir comme simple masse de manœuvre manipulée par les élites [...], [à] reconnaître [...] [sa] capacité d’initiative (agency) libre et souveraine [...], [à] redécouvrir sa culture propre, [à] s’intéresser enfin sérieusement à son univers de pensée et d'expérience (et pas seulement à ses conditions matérielles d'existence) » (Pouchepadass, $2000: 165)$. Ce sur quoi insiste cette perspective, c'est donc sur la capacité de vision et d'initiative des groupes dominés. Cette capacité est bâtie sur des valeurs, sur des normes et sur des affects. 
La perspective postcoloniale propose quant à elle une réflexion approfondie sur l'identité (Bhabha, 2007) inspirée d'Edward Said (2005) à partir de recherches qui trouvent leur origine dans la critique littéraire et dans l'anthropologie (Brisson, 2018 ; Collignon, 2007 ; Debonneville, 2017 ; Demart, 2016 ; Pouchepadass, 2007). Le projet postcolonial vise notamment à identifier ce qui survit du passé colonial dans les représentations sociales, entre autres dans les lois (Mendoza, 2011). Cette perspective est très attentive, à partir des travaux de Michel Foucault, aux formations discursives qui fondent les représentations hégémoniques du monde. Il s'agit, comme l'écrit Pouchepadass (2007), de critiquer une « configuration particulière du rapport entre savoir et pouvoir» (p.179). La perspective postcoloniale ouvre des horizons de réflexion sur les normes qui complètent bien, notamment en les replaçant dans une attention à la modernité, les analyses de Pierre Bourdieu (2012) sur l'État comme système de pensée et de pouvoir. Partant de l'idée que la colonisation a permis l'émergence du capitalisme (Mendoza, 2016), la perspective décoloniale développée par des chercheur·e·s d'Amérique latine et des Caraïbes (Demart, 2016) constitue une radicalisation de la réflexion.

Decolonial theorists emphasize that coloniality is different from colonialism. In contrast to the historically specific acts of colonialism through which one nation imposes its sovereignty on another, coloniality refers to long-standing patterns of power that emerge in the context of colonialism, which redefine culture, labor, intersubjective relations, aspirations of the self, common sense, and knowledge production in ways that accredit the superiority of the colonizer. Surviving long after colonialism has been overthrown, coloniality permeates consciousness and social relations in contemporary life. (Mendoza, $2016: 114)$

Cette perspective, qui part de l'idée que la colonialité du pouvoir se poursuit après les processus de décolonisation (Bermúdes, 2011 ; Giraldo, 2016), a pour ambition de décoloniser l'épistémologie contemporaine (Escobar, Restrepo, 2010 ; Mignolo, 2011 ; Quijano, 2000, 2007). C'est dès lors la question même du récit scientifique qui est posée par cette perspective, dans le but d'éviter de produire, comme l'écrit Ramón Grosfoguel (2007), « knowledge from the Western man "point zero" god-eye view» (p. 215). Breny Mendoza $(2002$; 2014) explique que la colonialité du pouvoir s'exerce dans la démocratie. Elle montre que la racialisation et le processus de différenciation sexuée des personnes non-européennes a permis la production de la citoyenneté blanche et mâle ainsi que la perpétuation des privilèges des hommes blancs malgré par exemple les garanties constitutionnelles concernant l'égalité formelle. En prendre conscience est essentiel pour notre objet, car les droits sociaux sont liés à un État qui propose un «point de vue institué en tant que point de vue légitime, c'est-à-dire en tant que point de vue que tout le monde doit reconnaître au moins dans les limites d'une société déterminée » (Bourdieu, $1987: 162$ ).

Ces trois perspectives, trop brièvement résumées ici, peuvent selon nous orienter la manière dont la critique peut être entendue empiriquement. Sur la base de ce que nous 
enseigne la perspective subalterne, ce sont les valeurs - notamment leur conception de la respectabilité (Skeggs, 2015) - les normes et les affects, qui sont en fait des « savoirs en pratique » (Connell, 2007; Connell, et al., 2017), qui sont intéressants à étudier. Mais comme l'écrit Howard Becker (2016), « obtenir des informations est toujours une négociation. [...] Face à nos tentatives pour obtenir d'eux ce que nous voulons, nos sujets d'étude ne sont pas forcément sans défense. Bien souvent, ils nous disent que cela ne les intéresse pas, qu'ils ne veulent pas se prêter à notre jeu, et ils s'en vont » (p. 178). Dans le cas du non-recours raisonné aux prestations sociales, cela signifie qu'un entretien avec des questions fermées ou semi-ouvertes sur le non-recours ne serait guère adapté, les personnes convoquant usuellement la rationalité dominante (manque d'information, dysfonctionnalité du système, stigmates, etc.) sans questionner le système même des droits sociaux. Nous proposons dès lors, comme l'écrit Dipesh Chakrabarty (1995), d'«"aller vers le subalterne", de "le laisser mettre en question nos conceptions de l'universel”, de faire leur place à l'affectif, au religieux, à “ce que nous avons fini, en devenant modernes, par voir comme irrationnel” »(Pouchepadass, 2000 : 176 sq.). Cela signifie au plan empirique qu'il faut non seulement un dispositif de recherche qui permette de s'investir dans un terrain, en prenant le temps de connaître les personnes rencontrées et d'échanger avec elles, mais également interroger notre propre rapport aux droits sociaux afin de ne pas imposer, fut-ce par inadvertance, notre point de vue aux personnes rencontrées. Nous devons en outre dans cette interaction garder en mémoire ce qu'enseigne la recherche : les décisions de non-recours sont souvent partielles et n'ont pas toujours un caractère permanent. Cette perspective se rapproche de celles prônées dans les recherches participatives ou partenariales en ce sens qu'elle est très attentive à ce que disent les personnes concernées de l'objet de recherche.

De la perspective postcoloniale, nous pouvons retenir l'attention qu'il est nécessaire de porter sur la manière dont le pouvoir s'incarne dans les contacts et les procédures administratives auxquelles les personnes sont confrontées dans le cadre de l'État social. Comme le non-recours raisonné est souvent partiel, temporaire et ponctuel, cela signifie en effet que les personnes ont été, ou sont encore en relation avec des dispositifs mettant en œuvre des droits sociaux. Elles ont été confrontées à des manières de concevoir le monde qui ne se pensent pas comme partiales, mais comme objectives et universelles, comme le fait de devoir décliner un âge, une nationalité, un sexe, une situation familiale, etc. Les réactions des personnes à cette obligation de faire coller une identité à une catégorie administrative nous semblent très intéressantes à recueillir via les récits qu'elles peuvent faire, une fois la confiance établie, de leur relation aux droits sociaux.

Enfin, la perspective décoloniale nous rappelle que nous parlons toujours depuis un emplacement particulier dans les structures de pouvoir, et que situer notre point de vue comme le propose l'épistémologie féministe (Haraway, 1988) signifie également situer les conditions de production de la recherche et raisonner sur les cadres épistémologiques que nous avons choisis, autrement dit sur l'«inconscient académique» 
(Clément et al., 2006). Il s'agit dès lors « au cours de l'enquête d'avoir "une meilleure maîtrise” du terrain et de l'objet à travers des détours réflexifs par les conditions de possibilité de la relation d'enquête, la subjectivité de chercheur ou de chercheuse, le paradoxe de l'observation, etc. » (Kocadost, 2017 : 29). La tenue d'un journal de terrain, une des méthodes de l'anthropologie, est bien sûr une manière classique de le faire ; il nous semble que l'écrire à partir d'une réflexion sur la colonialité du pouvoir offre de nouveaux outils pour réfléchir à cette relation. Le choix de nous doter d'outils qui ont fait leurs preuves dans d'autres contextes pour étudier le non-recours raisonné aux droits sociaux correspond en effet au projet de « critique générale des cadres de pensée traditionnels [...] des sciences sociales à partir de l'expérience des peuples déshérités » (Pouchepadass, 2004 : 74), et elle nous permet de réfléchir à partir d'autres cadres épistémologiques à la manière dont nous abordons nos terrains et aux analyses que nous produisons $^{8}$.

\section{Conclusion}

Dans cet article, nous sommes partis d'un angle mort de la théorie de la construction des problèmes sociaux, celui de la résistance des publics potentiels à la définition institutionnalisée des problèmes et de leurs solutions. Nous avons d'abord montré que la positivité des droits sociaux pour leurs publics potentiels a aujourd'hui un caractère hégémonique. Ensuite, nous nous sommes penchés sur la recherche sur le non-recours aux droits sociaux en relevant qu'il pouvait également résulter d'une non-demande, ou comme nous le disons avec des termes permettant de rendre compte de l'agentivité qui la motive, d'un raisonnement. Par hypothèse, ce non-recours raisonné est une critique furtive de la positivité des droits sociaux que nous cherchons à identifier empiriquement. Cette critique par des classes dominées est difficilement audible dans un contexte hégémonique, et c'est la raison pour laquelle nous proposons comme perspective de recherche de nous référer à des approches développées dans d'autres situations hégémoniques afin de trouver des outils pour l'entendre.

Le non-recours raisonné n'est donc selon notre perspective pas à considérer comme quelque chose d'exceptionnel, mais comme une critique sociale. Comme le suggère Jean-Paul Gagnon (2015), la critique est indispensable pour agir comme un sujet pensant en société. Or, l'identité des personnes qui recourent, recourent partiellement, ne recourent pas, de manière temporaire ou définitive, à des droits sociaux est influencée par la législation les concernant. Pour elles, à cause de leur situation, "the law [...] is "everywhere" in their lives » (Sarat, 1990 : 379). Un programme de recherche portant sur

\footnotetext{
${ }^{8}$ Les résultats de la recherche empirique que nous menons depuis 2017 seront présentés dans la thèse de doctorat que réalise la co-auteure de cet article, Frédérique Leresche dans le cadre du Pôle de recherche national LIVES - Surmonter la vulnérabilité : perspective du parcours de vie.
} 
l'étude de la critique furtive portée par le non-recours raisonné aux prestations sociales pourrait dès lors permettre de « rendre manifeste le décalage entre le monde social tel qu'il est et ce qu'il devrait être pour satisfaire aux attentes morales des personnes » (Boltanski, 2009: 56).

\section{Bibliographie}

Barbier J.-C., Letablier, M.-T. (dir.) (2008), Politiques sociales. Enjeux méthodologiques et épistémologiques des comparaisons internationales, Bruxelles, Peter Lang.

BECKER H. S. (2016), La bonne focale. De l'utilité des cas particuliers en sciences sociales, Paris, La Découverte.

BERMudes J. P. (2011), "Modernité/colonialité - décolonialité : une critique sociale autre », in M. MAESSCHALCK et A. LOUTE (dir.), Nouvelle critique sociale. Europe-Amérique Latine. Aller-Retour, Milano, Polimetrica, p. 195-231.

BEVERIDGe W. H. (1909), Unemployment, a Problem of Industry, London/New York/Bombay/Calcutta, Longsman, Green and Co.

Bнавна H. K. (2007), Les lieux de la culture. Une théorie postcoloniale, Paris, Payot.

Boltanski L. (1990), «Sociologie critique et sociologie de la critique », Politix, vol. 3, $\mathrm{n}^{\circ} 10-11, \mathrm{p} .124-134$.

Boltanski L. (2009), De la critique. Précis de sociologie de l'émancipation, Paris, Gallimard.

Bourdieu P. (1987), Choses dites, Paris, Minuit.

BOURDIEU P. (1993), La misère du monde, Paris, Seuil.

Bourdieu P. (2012), Sur l’État. Cours au Collège de France (1989-1992), Paris, Seuil.

BRISSON T. (2018), Décentrer l'Occident : les intellectuels postcoloniaux chinois, arabes et indiens et la critique de la modernité, Paris, La Découverte.

CAN GÜRCAN E., BAKMER O. (2015), « Post-neoliberal regional integration in Latin America: alianza bolivariana para los pueblos de nuestra América (ALBA) », in D. KREPS (dir.), Gramsci and Foucault: a reassessment, Farnham, Ashgate, p. 131-153.

Castles F. G., Leibfried S., Lewis J., Obinger H., Pierson C. (dir.) (2010), The Oxford handbook of the welfare state, New York, Oxford University Press.

CHAKRABARTY D. (1995), « Radical histories and question of enlightenment. Rationalism: some recent critiques of subaltern Studies ", Economic and political weekly, vol. 30, $n^{\circ} 14$, p. 751-759. 
Clement F., Roca i Escoda M., Schultheis F., Berclaz M. (2006), L'inconscient académique, Genève, Seismo.

Collignon B. (2007), « Note sur les fondements des postcolonial studies », EchoGéo, n 1. En ligne, consulté le 7 octobre 2019. URL : https://journals.openedition.org/echogeo/2089.

CONNELL R. (2007), Southern theory: the global dynamics of knowledge in social science, Crows Nest, Allen and Unwin.

Connell R., Collyer F., Maia J. O., Morrell R. (2017), « Toward a global sociology of knowledge: post-colonial realities and intellectual practices », International sociology, vol. 32, $\mathrm{n}^{\circ} 1$, p. 21-37.

DE CERTEAU M. (1990 [1980]), L’invention du quotidien. I. Arts de faire, Paris, Gallimard.

DebonNeVille J. (2017), «(Re)connaissances anthropologiques du postcolonial», Revue d'anthropologie des connaissances, vol. 11, n 3, p. 283-302.

DEMART S. (2016), « Au-delà de la controverse française : la critique postcoloniale dans le champ de la sociologie », SociologieS [En ligne], Dossiers, Sociétés en mouvement, sociologie en changement. En ligne, consulté le 22 février 2019. URL : https://journals.openedition.org/sociologies/5300

DeSROSIERES A. (1993), La politique des grands nombres. Histoire de la raison statistique, Paris, La Découverte.

Dubois H., LudwineK A. (2015), Access to social benefits: Reducing non-take-up, Luxembourg, Publications office of the European union (Eurofound).

Dubois V. (2012), « La promotion du contrôle : retour sur la construction politique de la fraude sociale comme problème public », in R. BoDIN (dir.), Les métamorphoses $d u$ contrôle social, Paris, La Dispute, p. 27-38.

ESCOBAR A., RESTREPo E. (2010), «Anthropologies hégémoniques et colonialité », Cahiers des Amériques latines, vol. 62, p. 83-95.

ESPING-ANDERSEN G. (1990), The Three Worlds of Welfare Capitalism, Princeton, Princeton university press.

ESPING-ANDERSEN G. (1999), Social foundations of postindustrial economies, Oxford, Oxford University Press.

EWick P., Silbey S. (1992), « Conformity, contestation and resistance: an account of legal consciousness », New England Law Review, vol. 26, p. 731-749.

EwICK P., SilBEy S. (1998), The Commonplace of Law. Stories of Everyday Life, Chicago, University of Chicago press. 
FASSIN D. (2009), « Les économies morales revisitées », Annales. Histoire, Sciences Sociales, vol. 64, n 6, p. 1237-1266.

FouCAult M. (1975), Surveiller et punir, Paris, Gallimard.

Foucault M. (1997), Il faut défendre la société. Cours au Collège de France (1976), Paris, Seuil/Gallimard.

Foucault M. (2001), Dits et écrits. Tome 2 : 1976-1988, Paris, Gallimard.

Fox PIVEN F., ClOWARD R. (1993 [1971]), Regulating the poor. The functions of public welfare, New York, Vintage Book.

FRAUENFELDER A., Togni C. (2007), « Les “abus” à l'aide sociale : une rhétorique au cœur du gouvernement de la misère », Carnets de bord en sciences humaines, vol. 13, p. 4355.

GAGNON J.-P. (2015), « Building a Gramsci-Foucault axis of democracy », in D. KREPS (dir.), Gramsci and Foucault: a reassessment, Farnham, Ashgate, p. 75-90.

Gilbert C., HenRy E. (dir.) (2009), Comment se construisent les problèmes de santé publique, Paris, La Découverte.

GILBERT C., HENRY E. (2012), « La définition des problèmes publics : entre publicité et discrétion », Revue française de sociologie, vol. 53, n 1, p. 35-59.

GIRALDO I. (2016), «Coloniality at work: decolonial critique and the postfeminist regime », Feminist Theory, vol. 17, n² 2, p. 157-173.

GoFFMAN E. (1952), «On cooling the mark out. Some aspects of adaptation to failure », Psychiatry, vol. XV, p. 451-463.

GRAMSCi A. (1978-1996), Cahiers de prison (vol. 5), Paris, Gallimard.

Grosfoguel R. N. (2007), « The epistemic decolonial turn », Cultural Studies, vol. 21, $\mathrm{n}^{\circ} 2$ 3, p. 211-223.

GuHA R. (1982), « On some apects of the historiography of colonial India », in R. GUHA (dir.), Subaltern Studies, vol. 1, Delhi, Oxford University Press, p. 1-9.

GuSFIELD J. R. (1980), The culture of public problems. Drinking-driving and the symbolic order, Chicago, The university of Chicago press.

HARAWAY D. (1988), "Situated knowledges: the science question in feminism and the privilege of partial perspective », Feminist Studies, vol. 14, n 3, p. 586-587.

HÜMBELIN O. (2016), Nichtbezug von Sozialhilfe und die Bedeutung von regionalen Unterschieden (Working paper), University of Bern. En ligne, consulté le 22 février 2019. URL : http://ideas.repec.org/p/bss/wpaper/21.html 
KNÜSEl R., Colombo A. (2014), «Accessibilité et non-recours aux services publics », Les politiques sociales, $\mathrm{n}^{\circ} 3$ et 4, p. 4-11.

Kocadost F. Ç. (2017), «Le positionnement intersectionnel comme pratique de recherche : faire avec les dynamiques de pouvoir entre femmes », Les cahiers $d u C E$ DREF, vol. 21, p. 17-50.

KORPI W. (2006), « Power resources and employer-centered approaches in explanations of welfare states and varieties of capitalism: protagonists, consenters, and antagonists », World politics, vol. 58, n² 2, p. 167-206.

KotT S. (2003), Bismarck, Paris, Presses de sciences PO.

LERESCHE F. (2019), "La critique portée par le non-recours aux droits sociaux: propositions pour développer une approche subalterne», SociologieS. En ligne, $\begin{array}{lllll}\text { consulté le } & 7 & \text { octobre } & 2019 . & \text { URL: }\end{array}$ https://journals.openedition.org/sociologies/11438.

LEWIS J. (1992), « Gender and the development of welfare regimes », Journal of european social policy, vol. 2, $\mathrm{n}^{\circ}$ 3, p. 159-173.

LEWIS J. (1997), «Gender and welfare regimes: further thoughts », Social Politics, vol. 4, $\mathrm{n}^{\circ} 2$, p. 160-177.

MARCHAND A. (2016), «Quand les cancers du travail échappent à la reconnaissance. Les facteurs du non-recours au droit », Sociétés contemporaines, vol. 102, n² 2, p. 103-128.

MARICHALAR P. (2017), Qui a tué les verriers de Givors ? Une enquête de sciences sociales, Paris, La Découverte.

Marshall T. H. (1996 [1950]), Citizenship and social class, in T. H. Marshall, T. BotTOMORE (dir.), Citizenship and social class, London and Chicago, Pluto Press, p. 1-51.

MAZET P. (2010), « La non demande de droits : prêtons l'oreille à l'inaudible », La vie des idées. En ligne, consulté le 22 février 2019. URL: https://laviedesidees.fr/La-non-demande-de-droits-pretons-l.html

MendozA B. (2002), "Transnational feminisms in question », Feminist Theory, vol. 3, $\mathrm{n}^{\circ} 3$, p. 295-314.

MEndozA B. (2011), « The role of the law in the rule of law of the new oligarchies: a latin american feminist perspective, feminists@law, vol. 1, nº 1, p.1-2.

Mendoza B. (2014), Ensayos de Crítica Feminista en Nuestra América, Mexico D.F., Editorial Herder.

MENDOZA B. (2016), « Coloniality of gender and power: from postcoloniality to decoloniality », in L. Disch, M. HAWKESWORTH (dir.), The Oxford handbook offeminist theory, New York, Oxford University Press, p. 100-121. 
MERRIEN F.-X. (2007), L'État-providence, Paris, PUF.

Mignolo W. (2011), « Géopolitique de la connaissance, colonialité du pouvoir et différence coloniale », Multitudes, vol. 6, n 3 , p. 56-71.

PeLISSE J. (2005), « A-t-on conscience du droit ? Autour des legal consciousness studies » Genèses, vol. 59, nº 2, p. 114-130.

Pierson C., Castles F. G., Naumann I. K. (dir.) (2014), The welfare state reader, Cambridge et Maiden, Polity press.

PouchePADASS J. (2000), « Les subaltern studies ou la critique postcoloniale de la modernité », L’Homme, vol. 156, p. 160-185.

POUCHEPADASS J. (2004), « Que reste-t-il des subaltern studies ? », Critique internationale, vol. 24, $\mathrm{n}^{\circ}$ 3, p. 67-79.

PouchePADASS J. (2007), «Le projet critique des postcolonial studies entre hier et demain », in M.-C. Smouts (dir.), La situation postcoloniale, Paris, Presses de Sciences Po, p. 173-217.

Quijano A. (2000), « Coloniality of power, ethnocentrism, and Latin America », Nepantla : views from South, vol. $1, \mathrm{n}^{\circ} 3, \mathrm{p}$. 533-580.

Quijano A. (2007), « "Race” et colonialité du pouvoir », Mouvements, vol. 51, n 3, p. 111118.

RAMAuX C. (2007), «Quelle théorie pour l'État social. Apports et limites de la référence assurantielle. Relire François Ewald 20 ans après », Revue française des affaires sociales, $\mathrm{n}^{\circ} 1, \mathrm{p} .13-34$.

Rosanvallon P. (1981), La crise de l'État-providence, Paris, Le Seuil.

SAID E. (2005), L'Orientalisme. L'Orient créé par l'Occident, Paris, Le Seuil.

SAinsbury D. (dir.) (1999), Gender and welfare state regimes, Oxford, Oxford Univ. Press.

Salais R., BaVerez N., Reynaud B. (1986), L'invention du chômage. Histoire et transformations d'une catégorie en France des années 1890 aux années 1980, Paris, PUF.

SARAT A. (1990), « “...The law is all over”: power, resistance and the legal consciousness of the welfare poor », Yale Journal of Law and Humanities, $n^{\circ} 2$, p. 343-379.

SCHULZKE M. (2015), « Power and resistance: linking Gramsci and Foucault », in D. KREPS (dir.), Gramsci and Foucault: a reassessment, Farnham, Ashgate, p. 57-73.

SkEgGs B. (2015), Des femmes respectables. Classe et genre en milieu populaire, Marseille, Agone. 
SMART B. (1989), « La politique de la vérité et le problème de l’hégémonie », in D. CouzENS Hoy (dir.), Michel Foucault - Lectures critiques, Bruxelles, Éditions universitaires et de Boeck, p. 177-194.

STONE-MEDiATORE S. (2003), Reading across borders: storytelling and knowledges of resistance, New York, Palgrave Macmillan.

TABIN J.-P. (1999), Les paradoxes de l'intégration. Essai sur le rôle de la non-intégration des étrangers pour l'intégration de la société nationale, Lausanne, Cahiers de l'éésp.

TABIN J.-P., Togni C. (2013), L'assurance chômage en Suisse. Une socio-histoire (1924-1982), Lausanne, Antipodes.

Titmuss R. (1969 [1958]), Essays on « The welfare state », London, Allen et Unwin.

Titmuss R. (1974), Social Policy. An introduction, London, Allen et Unwin.

Togni C. (2015), Le genre du chômage. Assurance chômage et division sexuée du travail en Suisse (1924-1982), Lausanne, Antipodes.

Topalov C. (1994), Naissance du chômeur, 1880-1910, Paris, Albin Michel.

Topalov C. (1999), Laboratoires du nouveau siècle. La nébuleuse réformatrice et ses réseaux en France, 1880-1914, Paris, EHESS.

VAN OORSCHOT W. (1991), " Non-take-up of social security benefits in Europe », Journal of european social policy, vol. $1, \mathrm{n}^{\circ} 1, \mathrm{p} .15-30$.

VAN OORSCHOT W. (1994), Take it or leave it. A study of non-take-up of social security benefits. Tilburg, Tilburg University Press.

VAN OORSCHOT W. (1995), Realizing rights - a multi level approach to non-take-up of meanstested benefits, London, Avebury.

VAN OORSCHOT W. (1996), «Les causes du non-recours : Des responsabilités largement partagées » Recherches et prévisions, vol. 43, nº 1, p. 33-49.

VIAL B. (2016), « Ne pas se sentir concerné par ses droits. Une analyse du non-recours dans les parcours sociaux juvéniles », Agora débats/jeunesses, vol. 74, n 3, p. 77-88.

WARIN P. (2006), L'accès aux droits sociaux, Grenoble, Presses Universitaires de Grenoble.

WARIN P. (2010), « Le non-recours : définition et typologies », Odenore, document de travail $\mathrm{n}^{\circ} 1$. En ligne, consulté le 22 février 2019. URL: https://odenore.mshalpes.fr/documents/WP1definition_typologies_non_recours.pdf

WARIN P. (2012), « Le non-recours aux droits », SociologieS [en ligne], Théories et recherches, En ligne, consulté le 7 octobre 2019. URL: https://journals.openedition.org/sociologies/4103. 
WARIN P. (2016), Le non-recours aux politiques sociales, Grenoble, PUG.

WARIN P. (2018), «Ce que demande la non-demande. Autour du non-recours aux aides sociales ", La vie des idées. En ligne, consulté le 22 février 2019. URL : https://laviedesidees.fr/Ce-que-demande-la-non-demande.html 